\title{
Creating Layout System in Prototype Less Production System
}

\author{
Yoshihiro Yamashita, Katutoshi Kanamori, and Hayato Ohwada
}

\begin{abstract}
Recently, the diversification of customer needs has progressed in the manufacturing industry. Therefore, it is required to create and examine layout at an early stage. In this study, we developed a system for examining the layout at the design phase. The layout created by the "Creating layout system" does not consider only efficient work but also easy work. We defined the non-placement space in order to eliminate the interference and parts boxes are placed considering its space. In addition, we proposed a way of creating layout. Creating layout system makes evaluation and 3DCG of created layout. It is possible to effective examine layout using "Creating layout system" early.
\end{abstract}

Index Terms-Creating layout system, 3DCAD, PLP system.

\section{INTRODUCTION}

Recently, short-lived the life of the product, the diversification of customer needs is in progress in the manufacturing industry. Therefore, in order to examine a proposed layout early, PLP (Prototype Less Production) has been proposed[1]. This production method allows you to create 3DCG (3D Computer Graphic) of assembly work from 3DCAD product data, to derive all feasible automatic assembly sequence, corresponding to the whole assembly sequence. Proposed the optimal assembly work by evaluating and analyzing them, rapid construction of production line is possible.

Previous studies of the layout in PLP, the method of determining the location of the parts box from the direction of the When installing the parts have been proposed [2]. These placements, for products that are composed of a few parts were effective. However, the problem when a large number of product parts, parts are placed sister to a position out of reach, that would have been derived infeasible proposed layout exists. Also, in order to adapt to the scene, it is necessary to consider not only efficiency, as well as the ease and simplicity of work to do.

The purpose of this study is to develop a system that can create and evaluate a proposed layout that can be applied to the field there. First of all, we define the cause of the impression given space to do the work in the actual workplace. By placing the parts box to non-space is defined, to create a layout of work in the impression given has been reduced. Then, for the purpose of efficiency and

Manuscript received September 12, 2012; revised November 7, 2012. This work was supported in Research Institute for Science and Technology in Tokyo University of Science.

Yoshihiro Yamashita with the Tokyo University of Science, Japan (e-mail: j7411631@ed. tus. ac. jp).

Katutoshi Kanamori and Hayato Ohwada are with the Tokyo University of Science, Japan. (e-mail: katsu@rs.tus.ac.jp; ohwada@ia.noda.tus.ac.jp). simplification of work, to experiment with six types of placements. In order to propose a variety of layouts, we propose and implement of systems to create layout.

\section{Conventional Problems}

3DCG is derived in this study is represented by VFDL (Virtual Factory Description Language). VFDL is a language and sentence structure represented by the detailed operation of the workers. Layout of the workshop, based on information in VFDL, is expressed in 3DCG (Fig. 1).

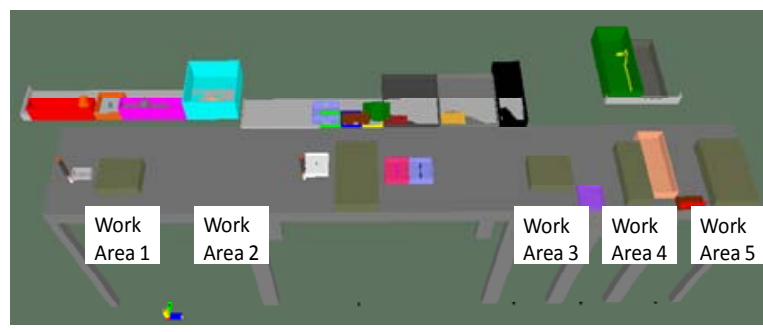

Fig. 1. Layout in the existing system.

As shown in Fig. 1, the workshop has been divided into the process for each fixture, the workshop has been built to place the fixtures and parts box to each process [2]. That is referred to as "work area" the workshop of each process was constructed here, side by side in one direction to go in order of use of a fixture each work area, it is possible to construct a production line type of a straight line. Production line, because it is composed of a set of work area, in order to create a good layout, you need to create a good work area.

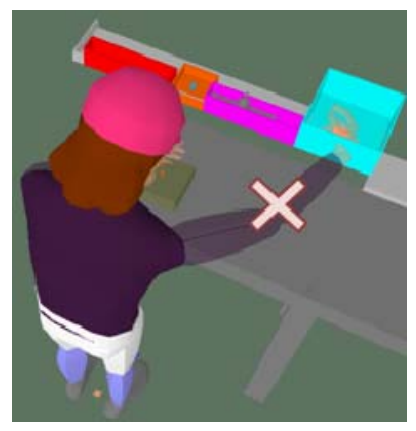

Fig. 2. Work area 1.

Work area was created in accordance with previous studies [2], which determine the position of the parts box from the direction to place the parts assembled. Parts box of parts from the parts box of parts assembled on the direction should be placed on a shelf, assembled from the left is placed to the left side of the fixture. If multiple parts box overlap is to move to the horizontal axis direction $\mathrm{Y}$, are doing the placement of the parts box so that they do not overlap all the parts box. I cannot even create a 3DCG and evaluation of the layout, make the 
appropriate layout of the proposed study. In addition, the proposed layout was created; it is desirable that the site is suitable for real. Therefore, it is necessary to determine the location of the parts box from the two sides of the ease of work and productivity.

\section{IDEA OF CREATING LAYOUT}

In this chapter, we describe the concept of creating the layout in order to solve the problems in Chapter 2. Assembly work in the present study, carried out by hand.

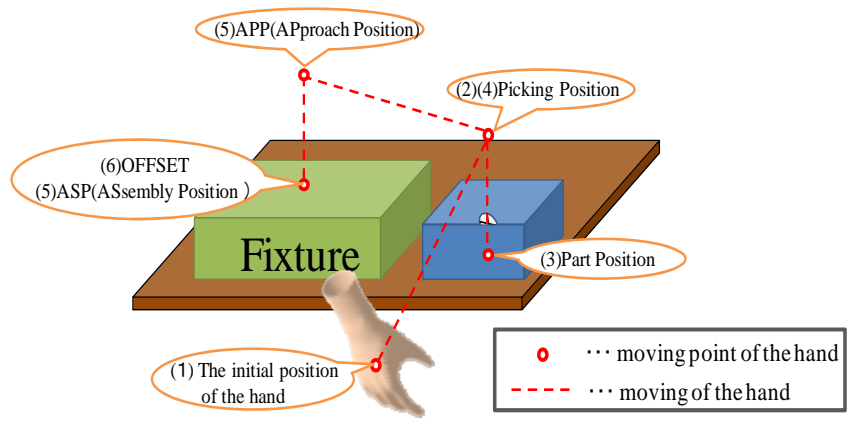

Fig. 3. Moving points of the hand at the time of assembly work.

General assembly work is carried out by the movement point of the hand of (1) to (6). (1)From the initial position of the hand, (2)go to the upper surface of the parts box, (3)grab the parts in the parts box (position of the part), (4)is removed from the parts box , (5)APP (APproach Position) or ASP(ASsembly Position) carry on, (6)assemble the parts(OFFSET). Later, repeated the flow of (2) to (6).

In other words, if we can make this kind of move smoothly, it is possible to perform efficient assembly work. However, there is a box that is placed components interfere with the movement of the hand. For example, if we can not verify the position of the parts, assembly work cannot be performed efficiently. I cannot do assembly and parts box has been placed into the space required for the picking and assembling of parts. In this study, these spaces is referred to as non-placement spaces, we defines Dead space, Picking space, of assembling space.

\section{HOW TO CREATE A LAYOUT}

In this section, we define the space that might interfere with the non-placement work first. Then, we describe how to deploy parts box.

\section{A. Non-Placement Space}

In this section, we describe the three non-placement space (Dead space, picking space, assembling space).

\section{1) Dead space}

Dead space is a space for workers to be present in the blind spot behind the fixture and parts box. When they entered the upper surface of the box to confirm the position of the component parts to perform within the dead space, hand movement $(2,3)$ is prevented. Therefore, the upper surface of the parts box to prevent from entering the dead space.

2) Picking space

$\mathrm{P}$ icking space is the space required when picking up the parts from the parts box. When you enter other things in this space, because the hand movement $(2,3,4)$ is prevented, you cannot pick up the parts. Therefore, picking space is created from the size of the hand [3] and the parts box , while others are not placed.

\section{3) Assembling space}

Assembling space is the space required for assembling the parts. When you enter other things in this space, because the hand movement is prevented, can not be assembled. Therefore, it is necessary to determine the space required for assembly work. Assembly space, when performing assembly work, is considered part passes through space(c-1) and hand passes space(c-2). As shown in Fig. 4 (left), assembly space(c-1) is created from the size of the parts and hand movement $(5,6)$. By adding the size of the hand in space, assembly space(c-2) is created assembling space. In addition, by performing the same for all parts to place, you can create a whole fixture assembling space (Fig. 4, right).
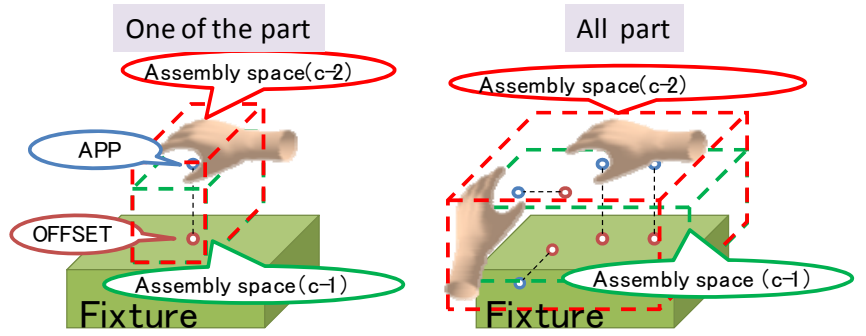

Fig. 4. Assembly space.

\section{B. How to Layout the Parts Box}

First of all, it is necessary to determine the area you want to place the parts box. To humans, there is a normal work area and the maximum work area [3] (Fig. 5). By placing these parts in the work area, the creation of a viable plan layout is possible. In addition, we consider to eliminate the occurrence of waste motion. One of the waste motions is giving the parts gripped by hand to the other hand. This behavior occurs when the hand of assembling parts is different from work area. placed parts. Therefore, it is divided into left and right around the work area. And, by placing parts box to work area of the assembling hand, it is possible to prevent the occurrence of giving the parts to other hand.
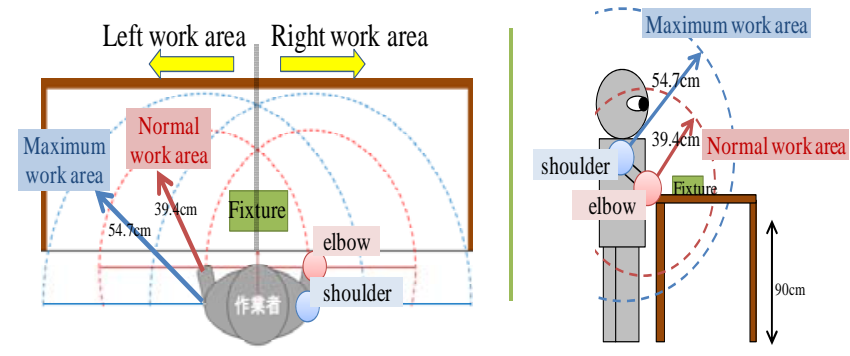

Fig. 5. Maximam and normal work area.

In this work area, while careful placement to non-space as defined in 4. 1, parts box is placed. When you do the placement of the parts box thinking only between different non-placement space do not overlap each other, there is a possibility to make a wasted space (Fig. 6). In this study, even if non-placement space overlapped, to place the parts box do not interfere with the movement of the hand there. For example, space (b) and (c-2) is a space for moving hand each other. Therefore, this does not preclude the movement of the hands that are overlapping each other as a space. In that case, 
the horizontal movement to the location of the $\mathrm{X}$-axis direction parts box so that it does not enter the space(c-1).

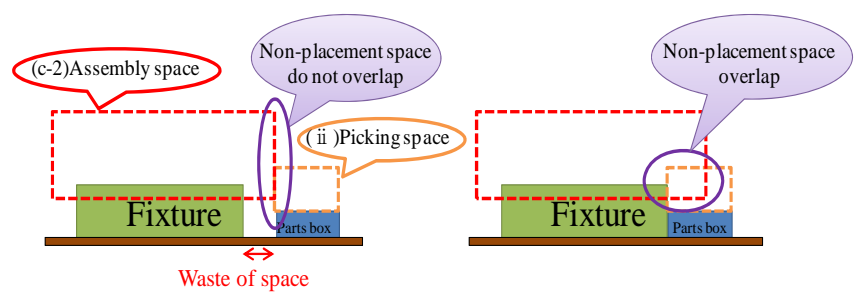

Fig. 6. Placement do not prevent the movement of the hand.

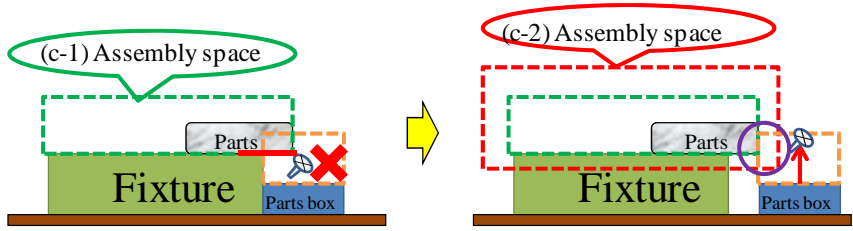

Fig. 7. Placement prevent the movement of the hand.

Parts box should be placed as shown in fig. 8 order to perform efficient assembly work. The first box of parts is located on a straight line with moving points of the hand (1) and (6). The second and subsequent box of parts are located in close proximity from a previous moving points of the hand (6). It is possible by this arrangement, the distance of moving points of the hand (1) and (6) are the shorter, more efficient to move the hand. However, in the case of a large number of product parts, in fact, they cannot be placed because they overlaps each other (Fig. 8). Therefore, it is necessary to consider how to place parts box in the defined area. In a general way, parts boxes are placed regularity in order to simplify the assembly work. Therefore, we consider the orientation and sequence the placement when you place the parts box. In this study, we placed in the direction of placement of the $\mathrm{X}$-axis direction and $\mathrm{Y}$-axis direction from the front workers. We experiment in three ways of placing sequence that are assembly sequence placement, parts box size placement, assembly position placement. Assembly sequence placement is a way to place the box on the sequence of the components used parts. Position of the parts easier to use then to recognize, worker use his hand smooth. Parts box size placement is a way to place the box on the sequence of the parts box has small bottom area. That can narrow between the parts box, it is possible to be closer moving hand point (4) to (5) and to reduce the distance of moving hand. Assembly position placement is a way to place sequence of the parts box that worker is closer to OFFSET position. It could potentially be used to smooth hands most when there are fewer parts. In this study, we experiment with six types of placements from the two orientations and the three sequences.

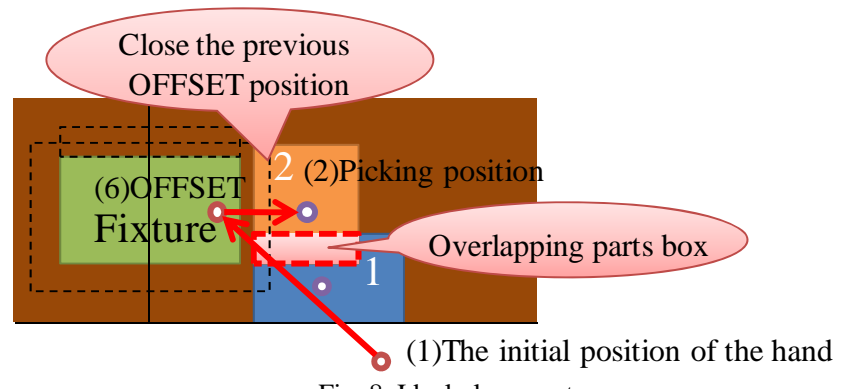

Fig. 8. Ideal placement.

Finally, we think about ways to shorten the walking distance and the moving distance of the hand. In order to minimize the distance of individual behavior, work area should be as small as not detrimental to the work [3]. As mentioned in 4 . 1 , there is a difficulty of the work space is clear. Therefore, it is possible to constrain the area using the limiting area line (Fig. 9). By placing the parts box so as not to exceed the limiting area line, the layout can be shortened walking distance and moving distance of the hand because moving hand point (4) approach moving hand point (5).

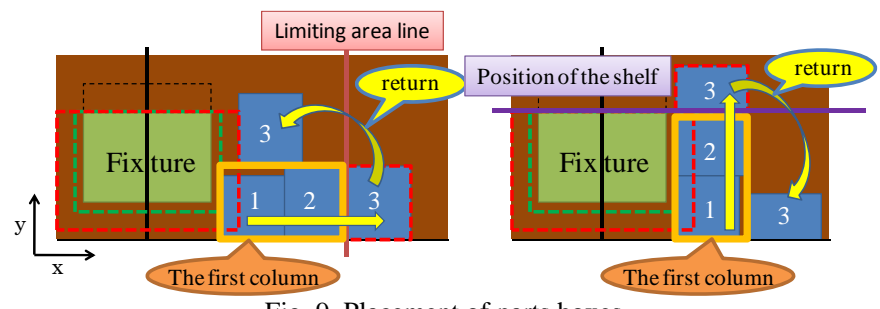

Fig. 9. Placement of parts boxes .

At the time, workplace is installed a shelf in order to put many parts box in limited work area. In addition, shelf is given the angle in order to look at locating the parts easier. The height of the shelf does not enter the space(c-2) and (b) and place behind the fixture because the space of desk and shelf is used effective.

\section{CReating LAyout SySTEM}

In this chapter, we describe the creating layout system that we developed based on the concept in Chapter 4. System was developed in this study is input VFDL_DATA described information of parts and VFDL that it don't be changed layout information. A new layout is created by creating and extracting the necessary information from VFDL_DATA and VFDL. Then, information of the operation of the workers are created so as to correspond to the new layout and evaluated.

\section{A. Create Assembly Space $(C-1)$ and (C2)}

First of all, creating layout system creates space (c-1) and (c-2). These spaces can be calculated from the size of the parts and hand, APP, OFFSET. It is necessary to divide VFDL into the work area because in order to create space(c-1) and (c-2) each fixture. In PLP, the worker performs the work always stood in front of the fixture. Therefore, worker always perform called "walk" when work area to perform work is changed. In addition, comparing the position of worker with the position of fixture, it is possible to determine whether he work which of the work area.

After the devide of VFDL, Creating layout system extracts the ASP and APP, OFFSET of all parts. Also, assembling Direction is determined from the change of coordinates of the ASP and APP, OFFSET. After that, it is possible to extract all the information needed to create the space(c-1) and (c-2) extracting sizes of parts corresponding parts number from VFDL_DATA. Space(c-1) and (c-2) are created based on the formulas (1) to (4). The formulas is used when parts is assembled from the upper direction that is occurred the most. As shown in Fig. 10, assembly space(c-1) and (c-2) is described by the coordinate of the position and sizes of space.

Formula (1) and (2) is used when calculating the coordinate of position and sizes of space in space(c-1). Formula (3) and (4) is used when calculating the coordinate 
of position and sizes of space in space(c-2). As these formulas, it is possible to calculate necessary space at the assembling work using information of sizes of parts and hand, difference of distance between position of APP and OFFSET. After the calculation of space for all parts, calculation results are converted the relative distance into the relative distance and we get the space(c-1) and (c-2) for each fixture.

$$
\begin{aligned}
& (x, y, z)=\left(\text { APP }_{x}-\text { PartsSize }_{x} / 2,\right. \\
& \text { APP } \left._{y}-\text { PartsSize }_{y} / 2, \text { APP }_{z}-\text { PartsSize }_{z} / 2\right) \\
& (x, y, z)=\left(\text { PartsSize }_{x}, \text { PartsSize }_{y},\right. \\
& \left.\left(\text { APP }_{z}-\text { OFFSET }_{z}\right)+\text { PartsSize }_{z}\right) . \\
& (x, y, z)=\left(\text { APP }_{x}-\text { PartsSize }_{x} / 2-53,\right. \\
& \left.A P P_{y}-\text { PartsSize }_{y} / 2-53, \text { APP }_{z}-\text { PartsSize }_{z} / 2\right) \\
& (x, y, z)=\left(\text { PartsSize }_{x}+106, \text { PartsSize }_{y}+106,\right. \\
& \left.\left(\text { APP }_{z}-\text { OFFSET }_{z}\right)+\text { PartsSize }_{z}+53\right) .
\end{aligned}
$$

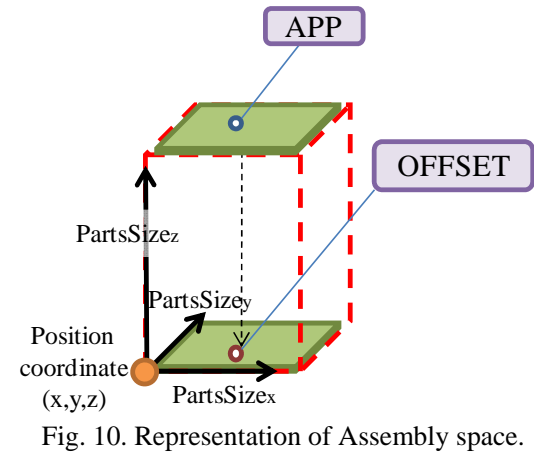

\section{B. Crate Layout}

Layout is created, based on the information that was created in space(c-1) and (c-2) in 5. 1 and VFDL. In addition, the parts will be placed in the core coordinate of the parts box.

\section{Process 1: Placement of fixture and desk}

Desk is placed $5 \mathrm{~cm}$ away from the operator. Fixture is placed $20 \mathrm{~cm}$ away from the operator because it is center of normal work area [3].

\section{Process 2: Determination of limiting area line}

The default value of limiting area line is sum of width of the most large parts box in the work area and space(c-2).

\section{Process 3: Determination of the placing sequence}

The parts is divided by hand used to assembly. After that, placing sequence is created by the sequence of the components used parts or the size of parts box or the position of OFFSET.

\section{Process 4: Placement of the first parts box}

The placement of the first parts box is determined by the space (c-1) and (c-2) and space of desk that is available placed. Candidate of a place to put the parts box is present in three places (F, S1, S2).

\section{(F)Front of the fixture}

The parts box is placed if the parts box does not protrude from the desk and does not enter space(c-1) and does not overlap space(c-2).

\section{(S1)Side of the fixture}

The parts box is placed if the parts box protrudes from the desk or enters space(c-1) and does not overlap space(c-2). At the time, if the parts box enters space(c-1), it is moved to the horizontal axis $\mathrm{X}$ due not to enter space(c-1).

(S2)Side of space(c-2)

If both conditions are not satisfied, the parts box is moved to horizontal axis $\mathrm{X}$ because of placing parts box not to overlap space(c-3).

\section{Process 5: Creating Non-placement space}

Non-placement space (a) and (b) is created from the size of the hand and parts box and the position placed parts box.

\section{Process 6: Placement of the second and subsequent parts box}

Nth parts box is placed to satisfy placing direction and not to protrude from the desk and to be next to N-1th parts box. However, placing position is selected as well as placement of the first one depending on the size of the parts box.

(S1') If the parts box does not overlap with the space(c-2)

The parts box is placed next to N-1th parts box. However, it is placed (b') as an exception if the parts box to enter space(c-1) because it prevent the movement of the hand.

(S2') If the parts box overlaps with the space(c-2)

If Nth parts box satisfies (b) of step 4, the parts box is moved to the location based on (4). Otherwise, it is moved to the location based on (c) of step 4 .

\section{Process 6-2: placement of stand}

The stand is established as necessary so that parts box placed in step 5 enter the space (a). If parts box placed in front is large, it is necessary to install the stand because the upper surface of the parts box enters space (a). The Height of the stand is determined by the difference between the height of the parts box placed. If the parts box placed in front is small, it is not necessary to install the stand because the upper surface of the parts box does not enter space (a). When you placed in step 6, if the Nth parts box is exceed the limiting space line or it exceed the position of the shelf, the position of parts box placed folded back so as to avoid exceeding these position(Fig. 9).

\section{Process 7: Placement of shelf}

If the position of the Nth parts exceeds the position of the shelf(y) or limiting area line, Nth parts box is placed on a shelf. The shelf is fixed to the angle of $45^{\circ}$ for easy to verify the position of the parts, the height of the shelf is the maximum value of space(c -2). After this Placement, the placement of the parts box continues to place next to the $\mathrm{N}-1$ th parts box on the shelf.

\section{Process 8: Replacement of parts box}

If the position of Nth parts box is placed on a shelf exceeds the limiting area line or it is greater than the maximum work area, the parts boxes is replaced. The limiting area line is larger than before, the new limiting area line is sum the previous limiting area plus the smallest size(x) of parts box. After that, all of the parts boxes are replaced until these boxes enter the work area through step 4 to 8 .

\section{Process 9: Determining of the size of desk}

From steps through 1 to 8, the placement of all fixtures and parts box has been completed. Therefore, it is possible to determine the size of the desk from the coordinate position of the parts box is located. The size of desk can be calculated from the difference between the value of minimum coordinate position of the parts box and the largest value of sum the coordinate of the parts box and the size of the parts box. 


\section{Process 10: Integration of the work area}

By repeating the number of fixture steps through 1 to 9 , all of the work area is created. Finally, the coordinates calculated by the relative coordinates with the fixture convert the absolute coordinates. The desks determined in step 9 translate and the parts boxes in each work area are translated to the same distance. In this way, it is possible to create a straight line layout.

\section{Synchronization of Layout and Behavior}

It is necessary to support the operation of the workers, so as to correspond to the newly created layout information. There is the anteroposterior relationship into the behavior written in VFDL. The behaviors of worker are formed to perform proper flow of this behavior.

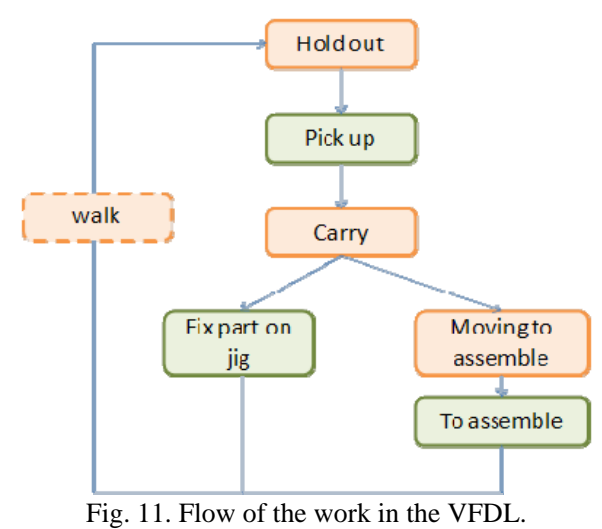

\section{Creating an Evaluation}

In this study, we evaluated the five rating scales, moving distance of the hand (mm), walking distance(mm), numbers of parts placed normal or maximum work area(pieces), numbers of giving the other hand a parts(pieces), the parts boxes placed ignoring sequence of the components used parts(number). It is possible to calculate the distance from change of each coordinate position. Determination of the work area, the position of the elbow and shoulder is assumed from a standing position of worker to calculate the distance between each parts. The regularity of the sequence assembly can be determined from the difference of the sequence of the parts box to place the order of the parts to be used.

\section{RESULT}

\section{A. Experimental product}

Creating layout system of the present study has been experimentally for product A of 23 parts and 19 parts box.

\section{B. Execution result}

The layouts of figure 12 to 14 were created by creating layout system. In the method of placement of all, without preventing the movement of the hand point that can take advantage of the space effectively includes the direction $\mathrm{z}$.

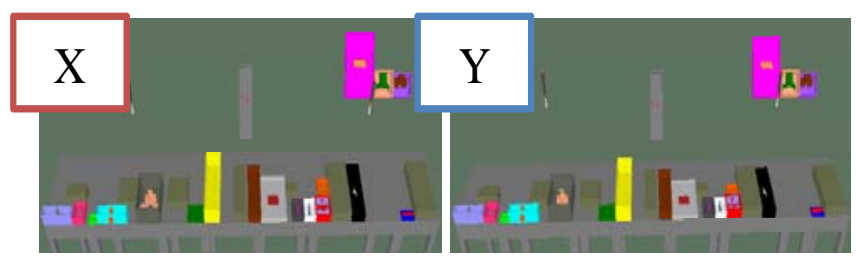

Fig. 12. Flow of the work in the VFDL.

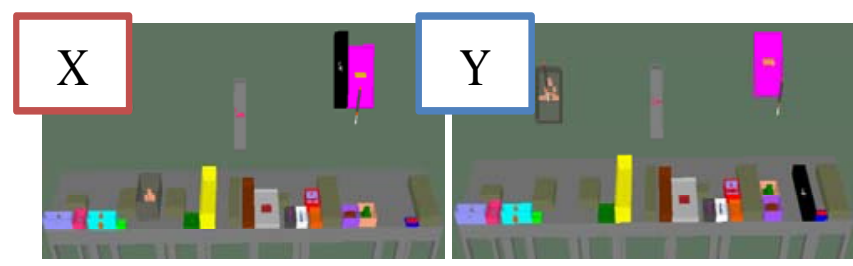

Fig. 13. Flow of the work in the VFDL.

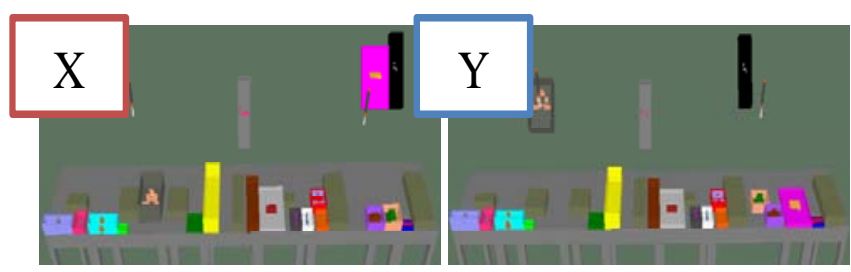

Fig. 14. Flow of the work in the VFDL.

TABLE I: EVALUATION OF THE PROPOSED LAYOUT

\begin{tabular}{|c|c|c|c|c|c|c|c|c|}
\hline \multirow{2}{*}{$\begin{array}{l}\text { direction } \\
\text { of } \\
\text { placement }\end{array}$} & \multirow{2}{*}{$\begin{array}{l}\text { placing } \\
\text { sequence }\end{array}$} & \multirow{2}{*}{$\begin{array}{c}\text { distance of } \\
\text { moving hand } \\
(\mathrm{mm})\end{array}$} & \multirow{2}{*}{\begin{tabular}{|} 
distance of \\
walk $(\mathrm{mm})$
\end{tabular}} & \multicolumn{3}{|c|}{\begin{tabular}{|c} 
numbers of parts in work area \\
(pieces)
\end{tabular}} & \multirow{2}{*}{$\begin{array}{l}\text { giving parts } \\
\text { the other hand } \\
\text { (number) }\end{array}$} & \multirow{2}{*}{$\begin{array}{c}\text { ignoring assembly } \\
\text { sequence } \\
\text { (number) }\end{array}$} \\
\hline & & & & \begin{tabular}{|c|} 
normal \\
work area \\
\end{tabular} & $\begin{array}{l}\text { maximum } \\
\text { work area }\end{array}$ & over & & \\
\hline \multirow{3}{*}{$X$-axis } & $\begin{array}{l}\text { assembly } \\
\text { sequence }\end{array}$ & 29435.7 & 5648.4 & 19 & 4 & 0 & 0 & 0 \\
\hline & $\begin{array}{c}\text { parts box size } \\
\text { placement }\end{array}$ & 30002.0 & 5793.5 & 20 & 2 & 1 & 0 & 6 \\
\hline & $\begin{array}{l}\text { assembly } \\
\text { position }\end{array}$ & 28293.7 & 5669.5 & 20 & 2 & 1 & 0 & 6 \\
\hline \multirow{3}{*}{ Y-axis } & $\begin{array}{l}\text { assembly } \\
\text { sequence }\end{array}$ & 29065.6 & 5648.4 & 19 & 4 & 0 & 0 & 0 \\
\hline & $\begin{array}{c}\text { parts box size } \\
\text { placement }\end{array}$ & 27809.1 & 5624.3 & 20 & 3 & 0 & 0 & 6 \\
\hline & $\begin{array}{l}\text { assembly } \\
\text { position }\end{array}$ & 28753.4 & 5747.3 & 20 & 2 & 1 & 0 & 6 \\
\hline
\end{tabular}

The results are shown in Table 1 were evaluated in each measure proposed layout was created. The proposed layout has been created in this study, many parts box are placed in the normal or maximum work area that is easily accessible of the hand. The layouts are evaluated from numbers of giving the other hand a parts and assembly sequence ignoring whether or not to utilize a smooth hand. The giving the other hand a parts has not occurred in the placement of all parts. So, it can take advantage of a smooth hand (Table1). Looking at the number of assembly sequence ignored, it has occurred numbers except by assembly sequence placement. The higher the number of assembly sequence ignored, assembly sequence do not be made out. Assembly sequence placement can take advantage of the hand most smoothly because the assembly sequence ignored does not occur. The parts box size placement creates efficient layout of the shortest distance of moving hand and walking distance when the waste space of between parts box are narrowed well. The assembly position placement is an efficient layout that is short distance of moving hand with a small number of parts such as this. In addition, the evaluation obtains better the Y-axis direction than $\mathrm{X}$-axis direction in the assembly sequence chosen in this study.

\section{CONCLUSION}

In this study, we defined the non-placement space that might interfere with the work from the point of hand movement and could propose to place parts boxes in order to 
perform the work easy and efficient. We presented six ways how to place parts boxes. These ways of placing the parts boxes could create automatically layouts that did not happen to give the parts the other hand and folded parts in maximum work area. It is possible to examine the production line using evaluation and 3DCG created by PLP system at the design phase.

\section{ACKNOWLEDGMENT}

We thank the Research Institute for Science and Technology in Tokyo University of Science for financial support.

\section{REFERENCES}

[1] Shingi Shinoda and Akira Niwa, "Study the basics of building a new production preparation process using data 3DCAD” IE review, vol. 44, No. 4, pp. 73-80, 2003.

[2] Akinori Kodera, "Proposal on how to create assembly line by 3DCG aimed at efficient and effective preparation for production", Tokyo University of Tokyo, 2009.

[3] Akira Masada, "ergonomics", 1997, The reference should be more than five items.

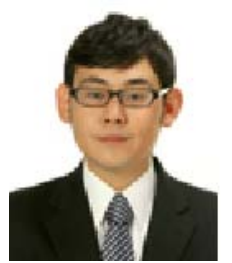

Yoshihiro Yamashita is university student, Tokyo University of Science. $\mathrm{He}$ is majoring in administrative engineering.

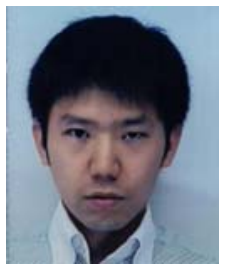

Katsutoshi Kanamori is Assistant Professor of Industrial Administration, Tokyo University of Science. He received Doctor of Science from Tokyo University of Science 2009. He has been working on Artificial Intelligence and Formulation of Creativity.

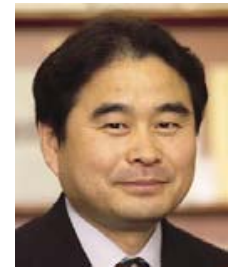

Hayato Ohwada is Professor of Industrial Administration and Director of Division of Next Generation Data Mining Technology, Tokyo University of Science. He received Doctor Engineering from Tokyo University of Science 1988. He has been working on Machine Learning and Inductive Logic Programming. 